related infection. ${ }^{1}$ The antiseptic-impregnated catheter was associated with a $44 \%$ reduction in catheter colonization (13.5 compared with 24.1 colonized catheters per 100 catheters; $P<.005$ ) and a $79 \%$ reduction in the rate of catheter-associated bloodstream infections (1.0 compared with 4.7 infections per 100 catheters).

Use of these antiseptic catheters also was associated with a significant reduction in the number of organisms colonizing the skin around the catheter insertion site. No adverse effects from the antiseptic catheter were seen, and none of the 122 isolates obtained from infected catheters in either group showed in vitro resistance to chlorhexidinesilver sulfadiazine. Antiseptic catheters also showed comparable benefit with catheters placed into a new site and catheters placed into an old site over a guidewire.

Cost-benefit analysis indicated that the antiseptic catheter should prove cost-beneficial if an institution's rate of catheter-related bacteremia with noncuffed CVCs is at least three infections per 1,000 catheter days.

In a second study, Dr. Issam Raad and colleagues from the Texas Medical Center Catheter Study Group conducted a multicenter, randomized clinical trial comparing triplelumen polyurethane CVCs pretreated with tridodecylmethyl-ammonium chloride and coated with minocycline and rifampin to untreated, uncoated catheters. ${ }^{2}$ The use of treated or coated catheters also was associated with significant reductions in the rates of catheter colonization and catheter-related bloodstream infections ( 0 bloodstream infections per 1,000 catheter days compared with 7.34 bloodstream infections per 1,000 catheter days). The authors did not report the effect of the coated catheters in reducing colonization of the catheter insertion site.

An important finding in both of these studies was that none of the impregnated catheters was associated with adverse events (hypersensitivity or toxicity) or infections caused by resistant pathogens; however, additional evaluation still is needed. Both studies conclude that the use of impregnated catheters, although more expensive than traditional catheters, result in a net cost savings.

In an accompanying editorial, Dr. Michele Pearson of the CDC and Dr. Elias Abrutyn of the Allegheny University of Health Science, Philadelphia, point out that, because these two studies addressed only a subset of CVCs-triple-lumen catheters-it remains to be determined whether the economic benefit would persist if the use of impregnated catheters was expanded to include CVCs that are used more commonly and have a lower risk for infection (for example, peripherally inserted CVCs, single-lumen CVCs, or tunneled $\mathrm{CVCs}$ for long-term use). ${ }^{3}$ They also note the importance of other measures to reduce risk of catheter-related infections, including the use of maximal barrier precautions (sterile gloves, large sterile drape; sterile gown, cap, and mask) for insertion of CVCs and use of skilled personnel for insertion and maintenance. They conclude that technological advances are an important advance in reducing the rate of CVC-related infections; however, their use should be viewed as an adjunct to, rather than a substitute for, good aseptic practices.

FROM: 1. Maki DG, Stolz SM, Wheeler S, Mermel LA. Prevention of central venous catheter-related blood- stream infection by the use of an antiseptic-impregnated catheter: a randomized, controlled trial. Ann Intern Med 1997;127:257-266.

2. Raad I, Darouiche R, Dupuis J, et al. Central venous catheters coated with minocycline and rifampin for the prevention of catheter-related colonization and bloodstream infections: a randomized, double-blind trial. Ann Intern Med 1997;127:267-274.

3. Pearson ML, Abrutyn E. Reducing the risk for catheter-related infections: a new strategy Ann Intern Med 1997;127:304-306.

\section{Sepsis Varies With Patient Population}

Sepsis syndrome is a leading cause of mortality in hospitalized patients. However, few studies have described the epidemiology of sepsis syndrome in a hospitalwide population. Researchers at the Channing Laboratory, Department of Medicine, Brigham and Women's Hospital, and Harvard Medical School in Boston conducted a prospective, multiinstitutional observational study including 5-month follow-up to describe the epidemiology of sepsis syndrome in the tertiary-care hospital setting. Eight academic tertiary-care centers were used, and each center monitored a weighted random sample of intensive-care unit (ICU) patients, non-ICU patients who had blood cultures drawn, and all patients who received a novel therapeutic agent or who died in an emergency department or ICU. Sepsis syndrome was defined as the presence of either a positive blood culture or the combination of fever, tachypnea, tachycardia, clinically suspected infection, and any one of seven confirmatory criteria. Estimates of total cases expected annually were extrapolated from the number of cases, the period of observation, and the sampling fraction. From January 4, 1993, to April 2, 1994, 12,759 patients were monitored, and 1,342 episodes of sepsis syndrome were documented. The weighted estimate of hospitalwide incidence of sepsis syndrome was 2.0 cases per 100 admissions, or 2.8 per 1,000 patient days. The unadjusted attack rate for sepsis syndrome between individual centers differed by as much as threefold, but, after adjustment for institutional differences in organ transplant populations, variation from the expected number of cases was reduced to twofold and was not statistically significant overall. Patients in ICUs accounted for $59 \%$ of total extrapolated cases, nonICU patients with positive blood cultures for $11 \%$, and nonICU patients with negative blood cultures for $30 \%$. Septic shock was present at onset of sepsis syndrome in $25 \%$ of patients. Bloodstream infection was documented in $28 \%$, with gram-positive organisms being the most frequent isolates. Mortality was $34 \%$ at 28 days and $45 \%$ at 5 months. It was concluded that sepsis syndrome is common in academic hospitals, although the overall rates vary considerably with the patient population. A substantial fraction of cases occur outside ICUs. The authors note that an understanding of the hospitalwide epidemiology of sepsis syndrome is vital for rational planning and treatment of hospitalized patients with sepsis syndrome, especially as new and expensive therapeutic agents become available. 
FROM: Sands KE, Bates DW, Lanken PN, et al. Epidemiology of sepsis syndrome in eight academic medical centers. Academic Medical Center Consortium Sepsis Project Working Group. JAMA 1997;278:234-240.

\section{PCR-A Cost-Effective Alternative for VRE Screening}

Surveillance cultures for vancomycin-resistant enterococci (VRE) are time-consuming and expensive for the laboratory to perform. In an effort to reduce the time of routine assays, investigators from the CDC's Hospital Infections Program conducted an extensive study on the use of polymerase chain reaction (PCR) as an alternative method of detecting and identifying VRE directly in fecal samples.

PCR primers, directed to $\operatorname{van} \mathrm{A}, \operatorname{van} B, \operatorname{van} C 1, \operatorname{van} \mathrm{C} 2$, and enterococcal ligase genes, were used to detect and identify VRE in fecal material obtained by rectal or perirectal swabbing. Although PCR-inhibitory substances were present in DNA prepared directly from the swabs, the inhibitory substances could be reduced by processing the nucleic acid with two commercially available DNA preparation columns. Cultures were grown on several selective agar media, before and after broth enrichment, from fecal material from 333 swabs. DNA was extracted from the fecal material and was analyzed by PCR. By using all four primer sets, only $59(67.8 \%)$ of the samples were positive for $\operatorname{vanA}$. However, after retesting the negative samples with only the vanA primer set, $77(88.5 \%)$ of 87 specimens that were culture-positive for Enterococcus faecium containing vanA were positive by PCR One specimen was PCR-positive for the vanA gene, but culture-negative for enterococci. The specificity of the vanA assay was $99.6 \%$. PCR analysis of enrichment broth samples with all four primers sets after 15 to 18 hours of incubation detected $74(85.1 \%)$ of the 87 culture-positive specimens. The specificity of the $\operatorname{van} \mathrm{A}$ assay after the enrichment step was $100 \%$. No vanB -containing enterococci were recovered by culture.

Because 16 samples can be tested by PCR in 4 hours (including electrophoresis), identification of VRE is possible within 8 hours of specimen submission at a cost of approximately $\$ 10.12$ per assay. The authors concluded that PCR may be a cost-effective alternative to culture for surveillance of VRE in some hospitals.

FROM: Satake S, Clark N, Rimland D, Nolte FS,
Tenover FC. Detection of vancomycin-resistant enterococci in fecal samples by PCR. J Clin Microbiol 1997;35:2325-2330

\section{National Guidelines Control Antibiotic Use -- Finland}

In the early 1990 s, there was an increase in erythromycin resistance among group A streptococci in Finland. In response, policies regarding outpatient antibiotic therapy were changed, and nationwide recommendations were issued that called for reductions in the use of macrolide antibiotics for respiratory and skin infections in outpatients. Investigators at the Antimicrobial Research Laboratory, National Public Health Institute, Turku, Finland, studied the effect of this national policy on the pattern of erythromycin resistance throughout Finland. Between 1991 and 1996, a total of 39,247 group A streptococcal isolates from throat swabs ( $82 \%$ of the isolates) and pus samples (18\%) and 290 isolates from blood cultures were studied in regional microbiology laboratories. The susceptibility of the isolates to erythromycin was tested by the disk-diffusion or the screening-plate method. Study results showed that consumption of macrolide antibiotics decreased from 2.40 defined daily doses, per 1,000 inhabitants per day in 1991 to 1.38 in $1992(P<.007)$ and remained near the lower level during the study period. The change in consumption was followed by a steady decrease in the frequency of erythromycin resistance among group A streptococcal isolates from throat swabs and pus samples, from $16.5 \%$ in 1992 to $8.6 \%$ in 1996 (odds ratio for 1996 as compared with 1992, 0.5; 95\% confidence interval, 0.4-0.5). The authors concluded that, in Finland, after nationwide reductions in the use of macrolide antibiotics for outpatient therapy, there was a significant decline in the frequency of erythromycin resistance among group A strep tococci isolated from throat swabs and pus samples.

FROM: Seppala H, Klaukka T, Vuopio-Varkila J, et al. The effect of changes in the consumption of macrolide antibiotics on erythromycin resistance in group A streptococci in Finland. Finnish Study Group for Antimicrobial Resistance. N Engl J Med 1997;337:441-446.

Additional news item in this issue: Outbreak of Mycoplasma Pneumonia, p 782. 